HU-SEFT R 1996-16,

FTUV 96/34, IFIC 96/40

(hep-ph/9606311)

\title{
DOUBLY CHARGED HIGGS AT LHC
}

\author{
K. HUITU \\ Research Institute for High Energy Physics \\ University of Helsinki, Finland \\ J. MAALAMPI \\ Department of Physics, Theory Division \\ University of Helsinki, Finland \\ A. PIETIL $\ddot{\mathrm{A}}$ \\ Department of Applied Physics \\ University of Turku, Finland \\ and \\ M. RAIDAL \\ Department of Theoretical Physics \\ University of Valencia, Spain
}

\begin{abstract}
We have investigated production of doubly charged Higgs particles $\Delta_{L, R}^{++}$via $W W$ fusion process in proton-proton collisions at LHC energies in the framework of the leftright symmetric model. The production cross section of the right-triplet Higgs $\Delta_{R}^{++}$is for representative values of model parameters at femtobarn level. The discovery reach depends on the mass of the right-handed gauge boson $W_{R}$. At best $\Delta_{R}^{++}$mass up to 2.4 $\mathrm{TeV}$ are achievable within one year run. For $\Delta_{L}^{++}$the corresponding limit is $1.75 \mathrm{TeV}$ which depends on the value of the left-triplet vev $v_{L}$. Comparison with Drell-Yan pair production processes shows that studies of the $W W$ fusion processes extend the discovery reach of LHC roughly by a factor of two. The main experimental signal of a produced $\Delta_{L, R}^{++}$ would be a hard same-sign lepton pair. There will be no substantial background due to the Standard Model (SM) interactions, since in the SM a same-sign lepton pair will always be associated with missing energy, i.e. neutrinos, due to lepton number conservation.
\end{abstract}




\section{Introduction}

Doubly charged scalar particles arise in many scenarios [1] extending the weak interactions beyond the Standard Model (SM). In the left-right symmetric (LR) electroweak theory [2] such a particle is a member of a triplet Higgs representation which plays a crucial part in the model. The gauge symmetry $S U(2)_{L} \times S U(2)_{R} \times U(1)_{B-L}$ of the LR model is broken to the SM symmetry $S U(2)_{L} \times U(1)_{Y}$ due to a triplet Higgs $\Delta_{R}$, whose neutral component acquires a non-vanishing expectation value in the vacuum. The $\Delta_{R}$, called the right-triplet, transforms according to $\Delta_{R}=(1,3,2)$, and it consists of the complex fields $\Delta_{R}^{0}, \Delta_{R}^{+}$and $\Delta_{R}^{++}$. If the Lagrangian is assumed to be invariant under a discrete $L \leftrightarrow R$ symmetry, it must contain, in addition to $\Delta_{R}$, also a left-triplet $\Delta_{L}=\left(\Delta_{L}^{0}, \Delta_{L}^{+}, \Delta_{L}^{++}\right)=(3,1,2)$. Hence the LR model predicts two kinds of doubly charged particles with different interactions. In contrast with $\Delta_{R}$, the existence of $\Delta_{L}$ is not essential from the point of view of the spontaneous symmetry breaking of the gauge symmetry. The vacuum expectation value $v_{L}$ of its neutral member is actually quite tightly bound by the $\rho$ parameter, i.e. by the measured mass ratio of the ordinary weak bosons.

The triplet Higgses have the following Yukawa couplings to the leptons:

$$
\mathcal{L}_{Y}=h_{R, i j} \Psi_{i R}^{T} C \sigma_{2} \Delta_{R} \Psi_{j R}+h_{L, i j} \Psi_{i L}^{T} C \sigma_{2} \Delta_{L} \Psi_{j L} \quad+\text { h.c. },
$$

where $\Psi_{i R, L}=\left(\nu_{i R, L}, l_{i R, L}\right)$ and $i, j$ are flavour indices. From the point of view of phenomenology a very important fact is that the $\mathrm{U}(1)_{B-L}$ symmetry prevents quarks from coupling to $\Delta_{R}$ and $\Delta_{L}$. In the processes that involve hadrons the triplet Higgses appear thus only through higher order corrections.

The Yukawa Lagrangian (1) leads to large Majorana mass terms of the form $h_{R, i j}\left\langle\Delta_{R}^{0}\right\rangle \nu_{i R} \nu_{j R}$ for the right-handed neutrinos. These give rise to the see-saw mechanism [3], which provides the simplest explanation to the lightness of ordinary neutrinos, if neutrinos do have a mass. The anomalies measured in the solar [4] and atmospheric [5] neutrino fluxes seem to require that neutrinos indeed have a mass, manifested in these phenomena through flavour oscillations. Furthermore, the observations of COBE satellite [6] may indicate the existence of a hot neutrino component in the dark matter of the Universe. In the framework of SM these observations are difficult to explain, since there neutrinos are predicted to be massless. 
Apart from the question of neutrino mass, the LR model is more satisfactory than the SM also in that it gives a better understanding of parity violation and it maintains the lepton-quark symmetry in weak interactions.

Nevertheless, so far there has been no direct evidence of left-right symmetry in weak interactions. This sets a lower bound to the energy scale of the breaking of that symmetry. According to the direct searches of the CDF and D0 experiments at Tevatron, the intermediate bosons of the right-handed interactions have the mass limits $M_{W_{R}} \gtrsim 652$ $\mathrm{GeV}$ [7] and $M_{W_{R}} \gtrsim 650 \mathrm{GeV}$ (720 GeV if the right-handed neutrino is assumed to be much lighter than $W_{R}$ ) [8], respectively. The mass limit for the new neutral intermediate boson is $M_{Z_{2}} \gtrsim 445 \mathrm{GeV}$ [9]. Although there are some assumptions behind these bounds concerning e.g. the strength $g_{R}$ of the right-handed gauge interactions in comparison with the strength $g_{L}$ of the left-handed interactions and the form of the CKM matrix of the right-handed interactions, which may, when relaxed, degrade the bounds considerably [10], it is reasonable to assume that below the scale of $0.5 \mathrm{TeV}$ the left-right symmetry is broken.

Hence, if the right-handed electroweak interactions exist, their discovery would require accelerators whose capacity exceeds that of the present ones. The phenomenological signatures of the LR model in $0.5-2 \mathrm{TeV}$ linear colliders have been recently under intensive study [11]. Particularly the signatures of the doubly charged scalar at $e^{+} e^{-}$ linear collider were discussed in ref. [12]. The production of a single doubly charged Higgs in ep collisions at Hera was studied in ref. [13].

In previous works only the pair production of the left-triplet $\Delta_{L}^{++}$in $p p$ collisions at SSC has been investigated [14]. In the present paper we shall investigate the prospects of testing the production of single doubly charged Higgs scalars in high-energy $p p$ collisions at LHC. Our main concern will be the right-triplet $\Delta_{R}^{++}$but we will also study the production of the left-triplet $\Delta_{L}^{++}$. Comparison with Drell-Yan pair production processes shows that the present study extends the discovery reach of $\Delta^{++}$at LHC considerably for most of the allowed parameter space of the model. The production of a single $\Delta_{L}^{++}$in $p p$ collisions was also investigated in ref. [15] in the framework of Georgi-Machacek model, which differs from the left-right symmetric model in some phenomenologically important points, e.g. it has a new complex triplet with $\mathrm{Y}=0$ and it is assumed that there are no Majorana type couplings of doubly charged Higgs to leptons.

Let us briefly summarize the present experimental limits on the mass $M_{\Delta}$ and the 
possibly non-diagonal couplings $h_{i j}$ of the doubly charged scalar particle (see [16] and references therein). The most stringent constraint comes from the upper limit for the flavour changing decay $\mu \rightarrow \bar{e} e e$ :

$$
h_{e \mu} h_{e e}<3.2 \times 10^{-11} \mathrm{GeV}^{-2} \cdot M_{\Delta^{++}}^{2} .
$$

From the Bhabha scattering cross section at SLAC and DESY the following bound on the $h_{e e}$ coupling was established:

$$
h_{e e}^{2} \lesssim 9.7 \times 10^{-6} \mathrm{GeV}^{-2} \cdot M_{\Delta^{++}}^{2}
$$

For $h_{e e}=0.6$, for example, the mass of the doubly charged boson should obey $M_{\Delta^{++}} \gtrsim 200$ $\mathrm{GeV}$, but for smaller couplings much lighter doubly charged scalars are still allowed. For the coupling $h_{\mu \mu}$ the extra contribution to $(g-2)_{\mu}$ yields the limit

$$
h_{\mu \mu}^{2} \lesssim 2.5 \cdot 10^{-5} \mathrm{GeV}^{-2} \cdot M_{\Delta^{++}}^{2}
$$

and the muonium transformation to antimuonium converts into a limit

$$
h_{e e} h_{\mu \mu} \lesssim 5.8 \cdot 10^{-5} \mathrm{GeV}^{-2} \cdot M_{\Delta++}^{2} .
$$

From non-observation of the decay $\mu \rightarrow e \gamma$ follows

$$
h_{e \mu} h_{\mu \mu} \lesssim 2 \cdot 10^{-10} \mathrm{GeV}^{-2} \cdot M_{\Delta^{++}}^{2} \text {. }
$$

From the condition of vacuum stability one can derive upper bounds on the couplings independent on the triplet Higgs mass [17]: $h_{e e}, h_{\mu \mu} \lesssim 1.2$.

The paper proceeds as follows. In the next section we will briefly describe the left-right symmetric model. Section 3 contains our results for the production and decay of $\Delta_{L, R}^{++}$, as well as a discussion on the background. Conclusions are presented in Section 4.

\section{Description of the left-right symmetric model}

In this section we will present the basic structure of the $S U(2)_{L} \times S U(2)_{R} \times U(1)_{B-L}$ leftright symmetric model. Quarks and leptons are assigned to the doublets of the gauge groups $S U(2)_{L}$ and $S U(2)_{R}$ according to their chirality:

$$
\begin{array}{cc}
\Psi_{L}=\left(\begin{array}{c}
\nu_{e} \\
e^{-}
\end{array}\right)_{L}=(2,1,-1), \quad \Psi_{R}=\left(\begin{array}{c}
\nu_{e} \\
e^{-}
\end{array}\right)_{R}=(1,2,-1), \\
Q_{L}=\left(\begin{array}{c}
u \\
d
\end{array}\right)_{L}=\left(2,1, \frac{1}{3}\right), \quad Q_{R}=\left(\begin{array}{c}
u \\
d
\end{array}\right)_{R}=\left(1,2, \frac{1}{3}\right),
\end{array}
$$


and similarly for the other families. The minimal set of fundamental scalars, if the theory is symmetric under the $L \leftrightarrow R$ transformation, consists of the following Higgs multiplets:

$$
\begin{gathered}
\Phi=\left(\begin{array}{cc}
\phi_{1}^{0} & \phi_{1}^{+} \\
\phi_{2}^{-} & \phi_{2}^{0}
\end{array}\right)=(2,2,0), \\
\Delta_{L}=\left(\begin{array}{cc}
\Delta_{L}^{+} & \sqrt{2} \Delta_{L}^{++} \\
\sqrt{2} \Delta_{L}^{0} & -\Delta_{L}^{+}
\end{array}\right)=(3,1,2), \\
\Delta_{R}=\left(\begin{array}{cc}
\Delta_{R}^{+} & \sqrt{2} \Delta_{R}^{++} \\
\sqrt{2} \Delta_{R}^{0} & -\Delta_{R}^{+}
\end{array}\right)=(1,3,2) .
\end{gathered}
$$

They transform according to $\Phi \rightarrow U_{L} \Phi U_{R}^{\dagger}, \Delta_{L} \rightarrow U_{L} \Delta_{L} U_{L}^{\dagger}$ and $\Delta_{R} \rightarrow U_{R} \Delta_{R} U_{R}^{\dagger}$, where $U_{L(R)}$ is an element of $S U(2)_{L(R)}$. The vacuum expectation value of the bidoublet $\Phi$ is given by

$$
\langle\Phi\rangle=\frac{1}{\sqrt{2}}\left(\begin{array}{cc}
\kappa_{1} & 0 \\
0 & \kappa_{2}
\end{array}\right) .
$$

This breaks the Standard Model symmetry $S U(2)_{L} \times U(1)_{Y}$. It generates masses to fermions through the Yukawa couplings $\bar{\Psi}_{L}^{i}\left(f_{i j} \Phi+g_{i j} \tilde{\Phi}\right) \Psi_{R}^{j}+h . c$. and $\bar{Q}_{L}^{i}\left(f_{i j}^{q} \Phi+g_{i j}^{q} \tilde{\Phi}\right) Q_{R}^{j}+$ h.c., where $\tilde{\Phi}=\sigma_{2} \Phi^{*} \sigma_{2}$.

The vacuum expectation values of the scalar triplets are denoted by

$$
\left\langle\Delta_{L, R}\right\rangle=\frac{1}{\sqrt{2}}\left(\begin{array}{cc}
0 & 0 \\
v_{L, R} & 0
\end{array}\right) .
$$

The right-triplet $\Delta_{R}$ breaks the $S U(2)_{R} \times U(1)_{B-L}$ symmetry to $U(1)_{Y}$, and at the same time the discrete $L \leftrightarrow R$ symmetry, and it yields a Majorana mass to the right-handed neutrinos, as was discussed in Introduction. It was also mentioned that the vacuum expectation value $v_{L}$ of the left-triplet is quite tightly bound by the $\rho$ parameter. One has $\left(\kappa^{2}=\kappa_{1}^{2}+\kappa_{2}^{2}\right)$

$$
\rho=\frac{M_{W_{L}}^{2}}{\cos ^{2} \theta_{W} M_{Z_{1}}^{2}} \simeq \frac{1+2 v_{L}^{2} / \kappa^{2}}{1+4 v_{L}^{2} / \kappa^{2}},
$$

and the experimental result $[9] \rho=1.0004 \pm 0.003$ then implies $v_{L} \lesssim 9 \mathrm{GeV}$, a small value compared with $\kappa \simeq 250 \mathrm{GeV}$.

The Higgs potential describing the mutual interactions of $\Phi, \Delta_{R}$ and $\Delta_{L}$ is in general quite complicated containing a great number of parameters. The most general potential for these fields is given in ref. [18]. There are severe constraints on the model parameters, the most crucial ones for our study concern $v_{L}$ and flavour changing neutral currents (FCNC). It was argued in ref. 18 that in phenomenologically consistent models either 
$v_{L}$ is exactly zero or a certain combination of the potential parameters, $\left(2 \rho_{1}-\rho_{2}\right)$, should vanish identically. Since there is no fundamental principle requiring $v_{L} \equiv 0$ we assume the latter possibility and study how to probe the value of $v_{L}$ in single $\Delta_{L}^{++}$production at LHC. In this case one expects the splitting between $\Delta_{L}^{++}$and $\Delta_{R}^{++}$masses to be large leading to the relatively light $\Delta_{L}^{++}$.

Unlike in the SM, in the LR model there are FCNC interactions mediated by some neutral Higgs fields which are certain superpositions of the neutrals members of the bidoublet $\Phi$. In order to suppress FCNC one must require the Higgs potential to be such that in the minimum $\kappa_{1} \ll \kappa_{2}$ or $\kappa_{1} \gg \kappa_{2}$. This requirement has the consequence that the $W_{L}, W_{R}$ mixing $\zeta$ is necessarily small, because $\zeta \simeq\left(g_{L} / g_{R}\right) 2\left|\kappa_{1} \kappa_{2}\right| /\left|v_{R}\right|^{2}$. We will assume $\kappa_{2}=0$, which leaves $W_{L}$ and $W_{R}$ as unmixed physical particles.

The masses of the charged gauge bosons are given in the case of no mixing and $v_{L}=0$ by the exact formulae

$$
\begin{aligned}
M_{W_{L}}^{2} & =\frac{1}{4} g_{L}^{2} \kappa_{1}^{2}, \\
M_{W_{R}}^{2} & =\frac{1}{4} g_{R}^{2}\left(2 v_{R}^{2}+\kappa_{1}^{2}\right) .
\end{aligned}
$$

If the $L \leftrightarrow R$ symmetry is implemented, the gauge couplings $g_{L}$ and $g_{R}$ should be equal $\left(g_{R}=g_{L} \simeq 0.64\right)$. If no such symmetry is assumed, the internal consistency within the model requires nevertheless $g_{R} \gtrsim 0.55 g_{L}$ [21]. In order to satisfy the lower mass limits of the new weak bosons $W_{R}$ and $Z_{R}$, the vev of the right-handed triplet, $v_{R}$, should be considerably larger than $\kappa_{1}$. Using the experimental value of the ordinary weak boson $M_{W_{L}}=81 \mathrm{GeV}$ and the Tevatron lower bound $M_{W_{R}} \gtrsim 650 \mathrm{GeV}$ and assuming $g_{R} \simeq g_{L}=0.64$, we find $v_{R} \gtrsim 5.6 \kappa_{1} \simeq 1.4 \mathrm{TeV}$.

The see-saw mass matrix of neutrinos is given by

$$
M=\left(\begin{array}{cc}
m_{L} & m_{D} \\
m_{D}^{T} & m_{R}
\end{array}\right) .
$$

The entries are $3 \times 3$ matrices given by $m_{D}=\left(f \kappa_{1}+g \kappa_{2}\right) / \sqrt{2}, m_{L}=h_{L} v_{L}$ and $m_{R}=h_{R} v_{R}$. The mass of the charged lepton is given by $m_{l}=\left(f \kappa_{2}+g \kappa_{1}\right) / \sqrt{2}$, and therefore if $f$ and $g$ are comparable, one has $m_{D} \simeq m_{l}$. Unless there is an extraordinary hierarchy among the couplings, one has $m_{L} \ll m_{D} \ll m_{R}$. In this case the approximate masses of the Majorana states that diagonalize the neutrino Lagrangian are given by $m_{\nu_{1}} \simeq m_{D}^{T} m_{R}^{-1} m_{D}$ and $m_{\nu_{2}} \simeq m_{R}$. 
Ignoring the mixing between families and considering the matrix (14) to present mixing of left-handed and right-handed neutrino of one family, one obtains the mass eigenvalues $m_{\nu_{1}} \simeq m_{D}^{2} / m_{R}$ and $m_{\nu_{2}} \simeq m_{R}$, and the mixing angle $\eta$ between these states is given by

$$
\tan 2 \eta=\frac{2 m_{D}}{m_{R}}
$$

The mixing of left- and right-handed neutrinos is thus in general very small, and one can identify $\nu_{1}$ with $\nu_{L}$ and $\nu_{2}$ with $\nu_{R}$ as a good approximation.

The mass of the heavier neutrino $\nu_{2}$, the "right-handed" neutrino, is related to the mass of $W_{R}$ via

$$
m_{\nu_{2}} \simeq \frac{h_{R}}{g_{R}} M_{W_{R}}
$$

Most naturally the heavy neutrino and the heavy weak boson would have roughly the same mass, but depending on the actual value of the Yukawa coupling constant $h_{R}$ the neutrino may also be lighter or somewhat heavier than $W_{R}$.

\section{Signals of doubly charged Higgs production at LHC}

\subsection{Production of doubly charged Higgses at LHC}

We will assume that the doubly charged Higgs bosons $\Delta_{L, R}^{++}$are light enough to be produced as real particles at LHC, and we will study separately their production and subsequent decay modes, concentrating mainly on the right-triplet $\Delta_{R}^{++}$. However, in the cases when the situation in the left-handed sector differs from the right-handed one we will present the differences. In $p p$-collisions $\Delta_{L, R}^{++}$cannot be produced in interactions of quarks because of the charge conservation. However, it can be produced through $W_{L, R} W_{L, R}$ fusion, either by virtual or real $W_{L, R}$ 's, depending on the mass of $\Delta_{L, R}^{++}$. The $W W$ fusion is not the only possibility, since also the physical singly charged Higgs scalars $\left(\kappa_{2}=0\right.$ assumed)

$$
\begin{aligned}
& h^{+}=\frac{1}{\sqrt{1+\frac{\kappa_{1}^{2}}{2 v_{R}^{2}}}}\left(\Phi_{1}^{+}+\frac{\kappa_{1}}{\sqrt{2} v_{R}} \Delta_{R}^{+}\right), \\
& \delta^{+}=\frac{1}{\sqrt{1+\frac{2 v_{L}^{2}}{\kappa_{1}^{2}}}}\left(\Delta_{L}^{+}+\frac{\sqrt{2} v_{L}}{\kappa_{1}} \Phi_{2}^{+}\right),
\end{aligned}
$$



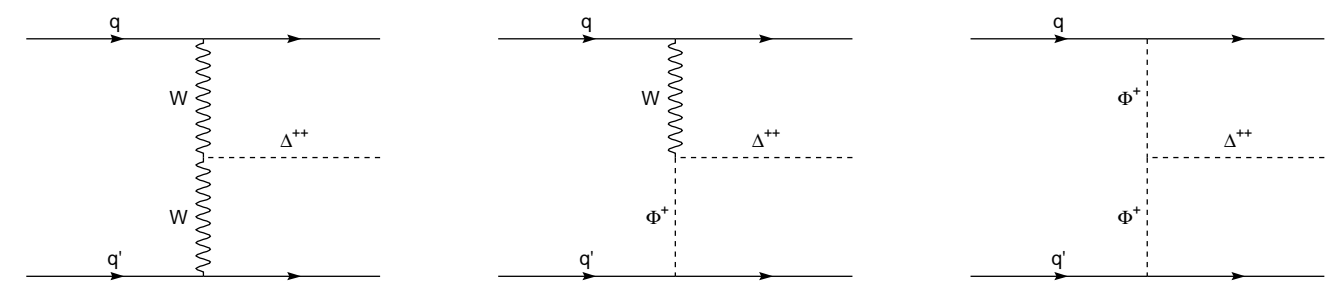

Figure 1: Feynman graphs contributing to the production of doubly charged Higgs at LHC.

have tree level couplings both to the doubly charged Higgses and the quarks.

Another production mechanism of $\Delta_{L, R}^{++}$at $p p$ collider is via $\gamma, Z_{L}, Z_{R}$ exchange, i.e. $q \bar{q} \rightarrow \gamma^{*}, Z_{R}^{*} \rightarrow \Delta_{R}^{++} \Delta_{R}^{--}$and $q \bar{q} \rightarrow \gamma^{*}, Z_{L}^{*} \rightarrow \Delta_{L}^{++} \Delta_{L}^{--}$. The latter process has been studied at SSC energy in ref. [14]. While for low values of $\Delta_{L, R}^{++}$mass the cross section of the pair production is comparable with, or may even exceed, that of the $W W$ fusion reactions, its kinematical reach is much lower. Indeed, we are going to see that with the present experimental constraints on model parameters the $\Delta_{L, R}^{++}$mass reach at LHC in the Drell-Yan processes is roughly half of the one in $W W$ fusion processes.

The Feynman graphs for the production processes of single $\Delta_{R}^{++}$are depicted in Fig.1, where $\phi^{+}$stands for the scalars $h^{+}$and $\delta^{+}$. Let us investigate the relative importance of the amplitudes. The $W W$ fusion vertex $W_{R}^{-} W_{R}^{-} \Delta_{R}^{++}$follows from the gauge invariant kinetic term $\left(D^{\mu} \Delta_{R}\right)^{\dagger} D_{\mu} \Delta_{R}$ as a result of the spontaneous breaking of the left-right symmetry, and it is given by

$$
\frac{1}{\sqrt{2}} g_{R}^{2} v_{R} W_{R}^{-} W_{R}^{-} \Delta_{R}^{++}
$$

The higher the symmetry breaking scale $v_{R}$ is, the stronger the coupling is, but on the other hand the heavier $W_{R}$ is. The corresponding vertex for the left-triplet $\Delta_{L}^{++}$is proportional to the small quantity $v_{L}$, which suppresses the fusion production of $\Delta_{L}^{++}$. However, in this case the lightness of $W_{L}$ boson enhances the production rate and, as explained in Section $2, \Delta_{L}^{++}$is expected to be somewhat lighter than $\Delta_{R}^{++}$. If $v_{L}=0$ then the Drell-Yan process is the only possibility to produce the left-triplet Higgs boson.

The $\phi^{-} \phi^{-} \Delta_{R}^{++}$coupling arises from the following quadratic terms of the scalar potential (we use the notation of ref. [18]):

$$
\beta_{1} \operatorname{Tr}\left(\Phi \Delta_{R} \Phi^{\dagger} \Delta_{L}^{\dagger}\right)+\beta_{2} \operatorname{Tr}\left(\tilde{\Phi} \Delta_{R} \Phi^{\dagger} \Delta_{L}^{\dagger}\right)+\beta_{3} \operatorname{Tr}\left(\Phi \Delta_{R} \tilde{\Phi}^{\dagger} \Delta_{L}^{\dagger}\right)
$$


which yields the couplings

$$
\frac{1}{\sqrt{2}} v_{L} \Delta_{R}^{++}\left(\beta_{1} \Phi_{1}^{-} \Phi_{2}^{-}-\beta_{2} \Phi_{1}^{-} \Phi_{1}^{-}-\beta_{3} \Phi_{2}^{-} \Phi_{2}^{-}\right)
$$

This contributes much less to the $\Delta_{R}^{++}$production than the $W W$ fusion (19) for several reasons. First, the dimensionful coupling $v_{L}$ is tiny compared with that of the $W_{R}^{-} W_{R}^{-} \Delta_{R}^{++}$ vertex $v_{R}$. Second, the couplings of the bidoublet fields $\Phi_{1}$ and $\Phi_{2}$ to quarks are Yukawa couplings and hence proportional to the mass ratio $m_{q} / M_{W_{L}}$, which is small compared with the gauge coupling $g_{R}$ appearing in the $W W$ fusion amplitude. Furthermore, in realistic models the coupling constants $\beta_{i}$ are necessarily small [18], otherwise one would face a serious fine tuning problem. In the case of $\delta^{+}$there is still an extra suppression due to the fact that the bidoublet component appears in $\delta^{+}$with just a small weight of $\sqrt{2} v_{L} / \kappa_{1} \lesssim 0.04$ (see Eq. (18)).

The $\Delta_{R}^{++} W_{R}^{-} \phi^{-}$vertex in the graphs of Fig.1. derives from the kinetic term $\left(D^{\mu} \Delta_{R}\right)^{\dagger} D_{\mu} \Delta_{R}$, which yields the coupling

$$
i g_{R} W_{R \mu}^{-}\left(\Delta_{R}^{-} \partial^{\mu} \Delta_{R}^{++}-\Delta_{R}^{++} \partial^{\mu} \Delta_{R}^{-}\right)
$$

This amplitude is possible for $h^{-}$, but not for $\delta^{-}$which does not have a right-triplet component. For $h^{-}$the process is suppressed, in addition to the small Higgs coupling with quarks, also due to the small weight $\kappa_{1} / \sqrt{2} v_{R}$ of the $\Delta_{R}^{-}$component.

We can conclude that the $W_{R} W_{R}$ fusion process dominates the production of single $\Delta_{R}^{++}$in $p p$ collisions, and the Higgs exchange diagrams can be safely ignored (except in the unprobable case that $W_{R}$ is orders of magnitude heavier than the singly charged Higgses). The same is also true for the left-triplet Higgs boson production process.

We have performed calculations of the $W W$ fusion process in Fig.1. without making any simplifying assumptions taking into account the three particle final state phase space. To obtain numerical values for the cross sections and final state distributions we have convoluted the functions over the initial state quarks momentum spectra using the default MRS-G set of the parton distributions of CERN Library program PDFLIB [19]. All numerical integrations have been performed by the integration routine VEGAS [20] which ensures high accuracy of the results.

The production cross sections for $\Delta_{L, R}^{++}$in $W W$ fusion are presented in Fig.2. by bold lines. In Fig.2. (I) the cross section of $\Delta_{R}^{++}$production is plotted for three different values of $W_{R}$ mass as a function of $\Delta_{R}^{++}$mass. For low $\Delta_{R}^{++}$masses the cross section is a quite 
rapidly falling function of $M_{W_{R}}$, e.g. going from $650 \mathrm{GeV}$ to $1.5 \mathrm{TeV}$, the cross section for $M_{\Delta_{R}}^{++}=200 \mathrm{GeV}$ decreases more than an order of magnitude. If light $\Delta_{R}^{++}$will be detected then this sensitivity can be used to obtain indirect information about $W_{R}$ mass. For heavy $\Delta_{R}^{++}$, however, the cross section depends on $M_{W_{R}}$ rather weakly which allows one to probe large Higgs masses. With the present lower value $M_{W_{R}}=650 \mathrm{GeV}$, the designed LHC luminosity of $100 \mathrm{fb}^{-1}$ per year and assuming that ten events are needed for discovery, $\Delta_{R}^{++}$as heavy as $2.4 \mathrm{TeV}$ can be found at LHC. We have also calculated the Drell-Yan pair production cross section of $\Delta_{R}^{++}$(assuming $Z_{R}$ to be very heavy) and presented it by dashed line in Fig.2. (I). For $M_{W_{R}} \lesssim 1 \mathrm{TeV}$ and $M_{\Delta_{R}}^{++} \gtrsim 500 \mathrm{GeV}$ the $W W$ fusion cross section exceeds the Drell-Yan one which falls below the discovery limit if $M_{\Delta_{R}}^{++} \gtrsim 1 \mathrm{TeV}$.

If $v_{L}$ vanishes, the production of $\Delta_{L}^{++}$in $W_{L}^{+} W_{L}^{+}$fusion is not possible due to the proportionality of the corresponding coupling to $v_{L}$ analogously to Eq. (19). The single production by other mechanisms is too small to be observed as explained previously. If $v_{L}$ differs from zero, also $\Delta_{L}^{++}$production is possible. For the allowed vev $v_{L}=9 \mathrm{GeV}$ the $W W$ fusion production cross section is shown in Fig.2. (II) by the bold line. In this case the free parameters to be tested are the vev $v_{L}$ and the mass of $\Delta_{L}^{++}$(the cross section scales as $v_{L}^{2}$ ). With the chosen value of $v_{L}$ the production cross section is comparable with the $\Delta_{R}^{++}$production one but it falls faster with the Higgs mass. The discovery limit of $\Delta_{L}^{++}$is as high as $1.75 \mathrm{TeV}$. In this case the Drell-Yan cross section, also presented in Fig.2. (II), is a few percent bigger than for the right-triplet Higgs due to additional $Z_{L}$ contribution but, as seen in figure, its discovery potential is the same.

Especially for background considerations it will be interesting to study the angular distribution of $\Delta_{R}^{++}$. In Fig. 3. the $p_{T}$ and $E_{\Delta_{R}}$ distributions are shown for $m_{\Delta_{R}^{++}}=500$ $\mathrm{GeV}$ and $M_{W_{R}}=1 \mathrm{TeV}$. Comparing the distributions it is seen that the doubly charged Higgses are mostly transverse. As the angular distribution of the decay products of $\Delta_{R}^{++}$ is flat, the loss of events in small forward angles is not essential.

\subsection{Decay of the triplet Higgs}

In the lowest order the doubly charged scalar $\Delta_{R}^{++}$can decay via the following channels:

$$
\begin{aligned}
\Delta_{R}^{++} & \rightarrow l^{+} l^{\prime+} \\
& \rightarrow W_{R}^{+} W_{R}^{+}
\end{aligned}
$$



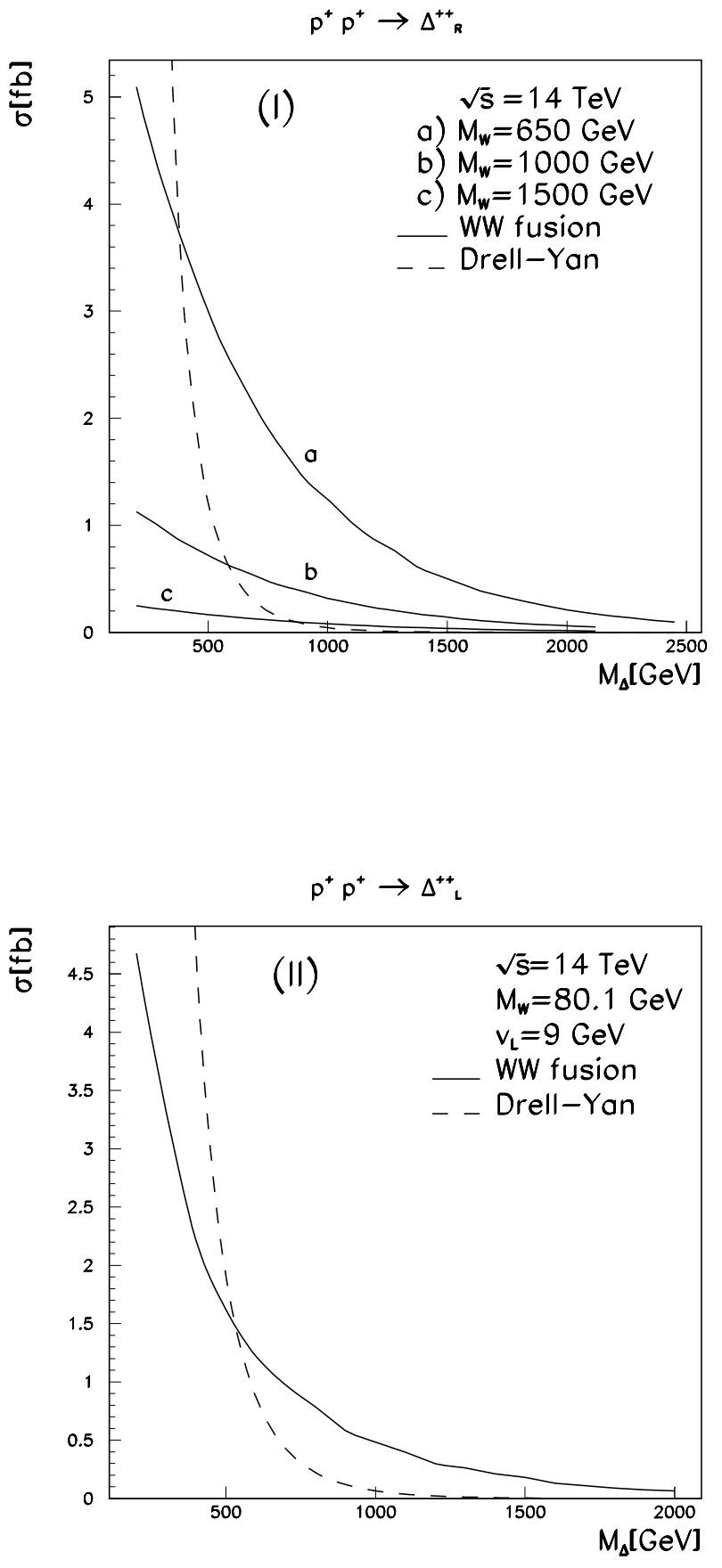

Figure 2: Production cross sections for $\Delta_{R, L}^{++}$as functions of doubly charged Higgs mass. 


$$
\begin{aligned}
& \rightarrow W_{R}^{+} h^{+}, \\
& \rightarrow W_{R}^{+} W_{R}^{+} \delta^{0}, \\
& \rightarrow h^{+} h^{+}, \\
& \rightarrow h^{+} h^{+} h^{0}, \\
& \rightarrow h^{+} h^{+} \delta^{0}, \\
& \rightarrow W_{R}^{+} h^{+} Z_{1}, \\
& \rightarrow W_{R}^{+} h^{+} Z_{2} .
\end{aligned}
$$

Here the neutral scalars $h^{0}$ and $\delta^{0}$ are mixtures of the real components of the states $\Phi_{1}^{0}$ and $\Delta_{R}^{0}$ so that in a good approximation $h^{0} \simeq \Phi_{1}^{0 r}$ and $\delta^{0} \simeq \Delta_{R}^{0 r}$. The charged Higgses $h^{+}$and $\delta^{+}$were defined in Eqs. (17) and (18).

Let us consider the relative magnitudes of the different decay channels. If there is a reasonable degeneracy between $\Delta_{R}^{++}$and the other members of the right-triplet, i.e. the singly charged scalar $h^{+}$having a right-triplet component and the predominantly righttriplet neutral scalar $\delta^{0}$, the modes (26) to (31) are likely to be kinematically suppressed or disallowed. The channel $\Delta_{R}^{++} \rightarrow W_{R}^{+} h^{+} Z_{1}$, while not necessarily kinematically suppressed, is in general quite small due to the smallness of $Z_{L}, Z_{R}$ mixing. This would leave us with the channels $\Delta_{R}^{++} \rightarrow l^{+} l^{\prime+}, \Delta_{R}^{++} \rightarrow W_{R}^{+} W_{R}^{+}, \Delta_{R}^{++} \rightarrow W_{R}^{+} h^{+}$.

Kinematically most favoured is the decay to a same-sign lepton pair, $\Delta_{R}^{++} \rightarrow l^{+} l^{\prime+}$, for which the decay width is given by

$$
\Gamma\left(\Delta_{R}^{++} \rightarrow l^{+} l^{\prime+}\right)=\frac{h_{R, l l^{\prime}}^{2}}{8 \pi} M_{\Delta_{R}^{++}} \simeq 4 \mathrm{GeV} \cdot h_{R, l l^{\prime}}^{2}\left(\frac{M_{\Delta_{R}^{++}}}{100 \mathrm{GeV}}\right)
$$

where the lepton masses are neglected. In Introduction the present bounds on the coupling constants $h_{R, l l^{\prime}}$ were given, and for the mass of $\Delta_{R}$ in the range we will assume it to lie, $M_{\Delta_{R}^{++}} \gtrsim 0.5 \mathrm{TeV}$, the diagonal couplings $h_{R, e e}, h_{R, \mu \mu}$ and $h_{R, \tau \tau}$ can according to these bounds be as large as $\mathrm{O}(1)$. In the case $l=l^{\prime}=e$ or $l=l^{\prime}=\mu$ we will have an unambiguous signature of a same-sign lepton pair with no missing energy. In the case $l=l^{\prime}=\tau$ the final state would include either two same-sign leptons $\left(e^{+} e^{+}, \mu^{+} \mu^{+}\right.$or $\left.e^{+} \mu^{+}\right)$ with missing energy or transverse pions plus missing energy. The even more spectacular signature of a same-sign electron-muon pair with no missing energy would be suppressed due to smallness of the non-diagonal coupling $h_{R, e \mu}$ as the bound (2) seems to indicate (or otherwise $h_{R, e e} \ll h_{R, e \mu}$ ). 
The decays $\Delta_{R}^{++} \rightarrow W_{R}^{+} W_{R}^{+}$and $\Delta_{R}^{++} \rightarrow W_{R}^{+} h^{+}$may also be kinematically disfavoured as the mass of the heavy boson $W_{R}$ is in general of the same order of magnitude as the mass of the Higgs triplet. If this is the case the decay to a like-sign lepton pair is the dominant mode in the lowest order. Let us, however, assume for the time being that $W_{R}$ is light enough for these processes to proceed and compare the magnitude of their widths with $\Gamma\left(\Delta_{R}^{++} \rightarrow l^{+} l^{\prime+}\right)$.

The rate of $\Delta_{R}^{++} \rightarrow W_{R}^{+} W_{R}^{+}$is given by

$$
\begin{gathered}
\Gamma\left(\Delta_{R}^{++} \rightarrow W_{R}^{+} W_{R}^{+}\right)=\frac{g_{R}^{2} M_{\Delta_{R}^{++}}}{8 \pi} \quad \sqrt{1-4\left(\frac{M_{W_{R}}}{M_{\Delta++}}\right)^{2}}\left(1-\left(\frac{M_{W_{L}}}{M_{W_{R}}}\right)^{2}\right)\left(3 \frac{M_{W_{R}}^{2}}{M_{\Delta^{++}}^{2}}\right. \\
\left.+\frac{M_{\Delta++}^{2}}{4 M_{W_{R}}^{2}}-1\right) .
\end{gathered}
$$

The $\Delta_{R}^{++} W_{R}^{-} W_{R}^{-}$vertex is proportional to the vev $v_{R}$, and thus this decay channel need not be suppressed like the corresponding channel of the left-triplet, where the coupling is proportional to the small or possibly vanishing vev $v_{L}$ of $\Delta_{L}^{0}$. Actually, for $M_{W_{R}}=0.5$ $\mathrm{TeV}$, the branching ratio of $\Delta_{R}^{++} \rightarrow W_{R}^{+} W_{R}^{+}$exceeds that of $\Delta_{R}^{++} \rightarrow l^{+} l^{+}$when $M_{\Delta^{++}} \gtrsim 2$ $\mathrm{TeV}$, assuming that the Majorana coupling $h_{R, l l}$ is of the order of one. If the energy of $\Delta_{R}^{++}$is not high enough for decay into a pair of real $W_{R}^{+}$'s, a decay channel including one or two virtual $W_{R}^{+}$'s might still be important. We have studied this possibility, but the lepton channel is then the dominating one, unless it is for some reason totally suppressed. If $h_{R, l l^{\prime}} \sim 0$, the branching ratio to virtual $W_{R}^{+}$'s is close to the branching ratio of $\Delta_{R}^{++} \rightarrow W_{R}^{+} h^{+}$.

The detection signal of the decay $\Delta_{R}^{++} \rightarrow W_{R}^{+} W_{R}^{+}$would be a hard like-sign lepton pair with missing energy, assuming that the two neutrinos produced along with the charged leptons are the light Majorana neutrinos $\nu_{1}$ or heavy Majorana neutrinos $\nu_{2}$ with a mass $m_{2} \ll M_{W_{R}}$. It can be distinguished from the similar final state of the $\Delta_{R}^{++} \rightarrow \tau^{+} \tau^{+}$ channel on grounds of its different angular distribution. If the heavy neutrino produced in the $W_{R}$ decay has a large mass, it will decay in the detector via $\nu_{2} \rightarrow l W_{R}^{*} \rightarrow l+$ two jets. The decay products are likely to be well separated, but nevertheless in the LHC environment this would constitute a much less background free signal than purely leptonic final states. The same is true for the case where $W_{R}$ 's are reconstructed in hadronic channels due to $W_{R} \rightarrow$ two jets.

Note that for non-vanishing $v_{L}$ the decay channel $\Delta_{L}^{++} \rightarrow W_{L}^{+} W_{L}^{+}$can be the dominant one for the left-triplet Higgs $\Delta_{L}$. In this case one has to tag four jets coming from $W_{L}$ decays and reconstruct the invariant mass of $\Delta_{L}^{++}$. 
The decay width of $\Delta_{R}^{++} \rightarrow W_{R}^{+} h^{+}$is given by

$$
\Gamma\left(\Delta_{R}^{++} \rightarrow W_{R}^{+} h^{+}\right)=\frac{g_{R}^{2} M_{\Delta_{R}^{++}}}{16 \pi} \frac{M_{W_{L}}^{2} M_{\Delta_{R}^{++}}^{2}}{M_{W_{R}}^{4}} \lambda^{3 / 2}\left(1,\left(\frac{M_{W_{R}}}{M_{\Delta_{R}^{++}}}\right)^{2},\left(\frac{M_{h}}{M_{W_{R}}}\right)^{2}\right),
$$

where $\lambda$ is the usual phase space suppression function. For small values of the $\Delta_{R}^{++}$mass, this width is considerably smaller than the width of the lepton pair channel, assuming that $h^{+}$has a mass of the same order of magnitude as the mass of $W_{R}$ or larger. The $h^{+}$ scalar decays via its $\Delta_{R}^{+}$component to $l_{R}^{+} \nu_{R}$ and via its $\Phi^{+}$component mainly to a top quark-antiquark pair.

To gain an idea of the relative magnitudes of the widths of the three main decay modes discussed above, consider some representative cases. For $M_{\Delta_{R}^{++}}=1 \mathrm{TeV}, M_{W_{R}}=0.5$ $\mathrm{TeV}, M_{h}=0.2 \mathrm{TeV}$ and $m_{\nu_{2}}=1 \mathrm{TeV}$ one has $\Gamma\left(\Delta_{R}^{++} \rightarrow l^{+} l^{\prime+}\right)=13 \mathrm{GeV}, \Gamma\left(\Delta_{R}^{++} \rightarrow\right.$ $\left.W_{R}^{+} W_{R}^{+}\right)=0 \mathrm{GeV}$ and $\Gamma\left(\Delta_{R}^{++} \rightarrow W_{R}^{+} h^{+}\right)=0.3 \mathrm{GeV}$. For $M_{\Delta_{R}^{++}}=2 \mathrm{TeV}, M_{W_{R}}=0.5$ $\mathrm{TeV}, M_{h}=0.5 \mathrm{TeV}$ and $m_{\nu_{2}}=1 \mathrm{TeV}$ the widths are $80 \mathrm{GeV}, 90 \mathrm{GeV}$ and $4 \mathrm{GeV}$, respectively.

In the following we will consider more closely the detection of $\Delta_{R}^{++}$via its leptonic decay and discuss the background from SM sources.

\subsection{Backgrounds from the Standard Model and the Left- Right Model}

We shall argue that a like-sign lepton pair as an experimental signal of $\Delta_{R}^{++}$production has a very low background from other processes. If the pair of like-sign electrons or muons is seen, as is the case with relatively light or very heavy $\Delta_{L, R}^{++}$'s (Fig.3.), the amount of missing transverse energy is tiny. Since all possible background channels from the SM contain missing energy due to neutrinos [22] in order to preserve lepton number, there is no background from the SM in the events of interest.

In the case of the left-right model, the background of the same order in perturbation theory is the "neutrinoless double beta decay" process, in which two same sign $W_{R}$ 's fuse to a like-sign lepton pair via exchanging a Majorana neutrino in t-channel. Using EPA distribution for longitudinal and transverse distributions of $W_{R}$ 's [23] inside colliding protons, we calculated the upper limit for this background. Although the contribution from individual process is larger if the $W_{R}$ is longitudinal [24], the beams contain mostly transverse gauge bosons [23]. It turns out that the contribution from the transverse $W_{R}$ 's 

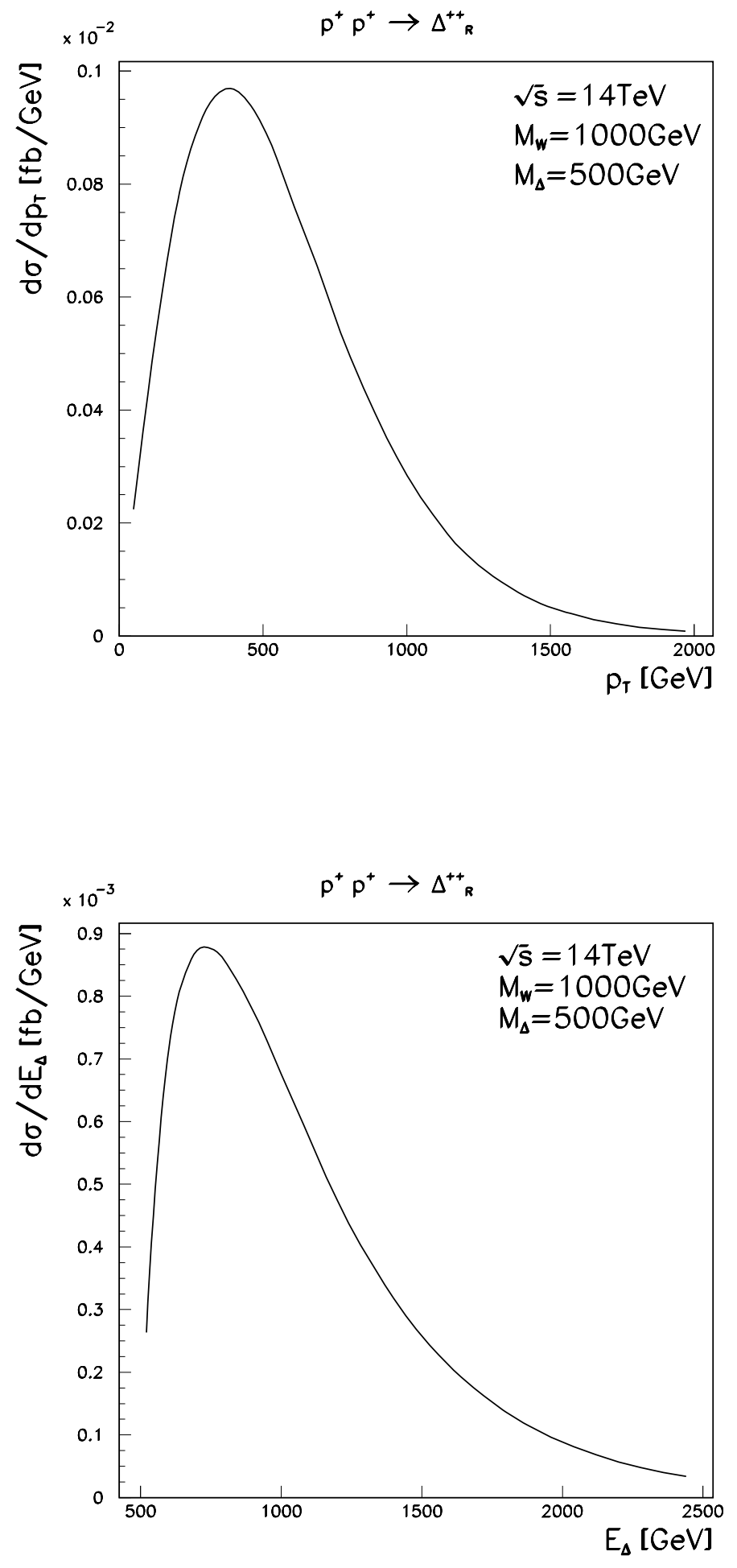

Figure 3: Distributions of $p_{T}$ and $E_{\Delta_{R}}$ for doubly charged Higgs production. 


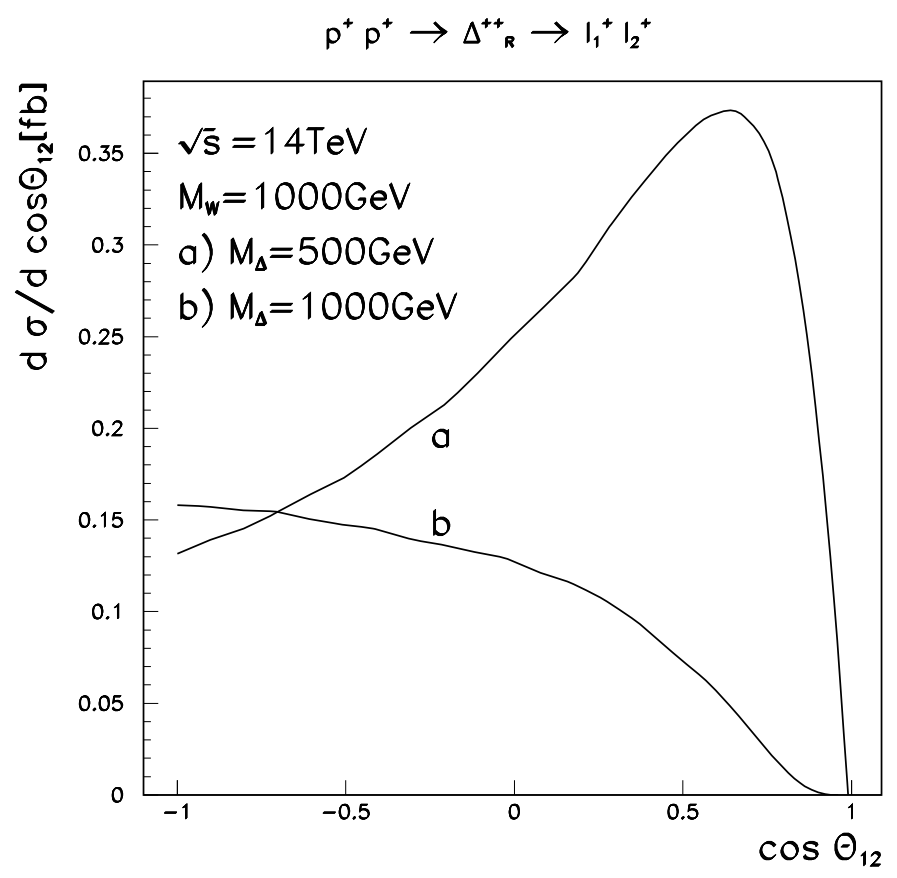

Figure 4: Opening angle distribution between leptons in $p p \rightarrow \Delta_{R}^{++} \rightarrow l^{+} l^{+}$in the $p p$ center of mass frame.

is the dominant one. For the masses we have used, the neutrinoless double beta decay contribution is $\mathcal{O}\left(10^{-4}\right) \mathrm{fb}$. This is negligible compared with the signal cross section.

The mass of the doubly charged Higgs can be reconstructed from the same charge leptons in the signal cases. As discussed in the previous section, the peak in the case of leptonic decay modes is relatively narrow (for $M_{\Delta_{R}^{++}}=1 \mathrm{TeV}$ and $M_{W_{R}}=0.5 \mathrm{TeV}$ one has typically $\left.\Gamma\left(\Delta_{R}^{++} \rightarrow l^{+} l^{\prime+}\right)=\mathcal{O}(10) \mathrm{GeV}\right)$. Thus it should be clearly visible in the signal cases.

Let us finally note that the opening angle between the two leptons strongly depends on the mass of $\Delta_{R}^{++}$, as is demonstrated in Fig. 4. In the case of a small mass $\Delta_{R}^{++}$'s are produced in average with a larger three-momentum than in the case of a large mass, making the decay products more boosted. 


\section{Conclusions and discussion}

We have studied the single production of doubly charged scalars $\Delta_{L, R}^{++}$through $W_{L, R}^{+} W_{L, R}^{+}$ fusion in $p p$ collisions at LHC in the framework of the left-right symmetric electroweak model. Since the relevant couplings involved are rather general, most of our results are also applicable in other models with scalar bileptons. The production cross section of the right-triplet Higgs $\Delta_{R}^{++}$depends on its mass as well as on the mass of $W_{R}$. An interesting feature of the cross section is that the $W_{R}$ mass dependence is relatively weak for large $M_{\Delta_{R}^{++}}$which allows one to probe large Higgs masses. For representative mass ranges 200 $\mathrm{GeV} \lesssim M_{\Delta_{R}^{++}} \lesssim 1000 \mathrm{GeV}$ and $650 \mathrm{GeV} \lesssim M_{W_{R}^{++}} \lesssim 1000 \mathrm{GeV}$ the cross section is 0.3 to $5 \mathrm{fb}$. With the anticipated LHC luminosity of $10^{34} \mathrm{~cm}^{-2} \mathrm{~s}^{-1}$ this would mean of the order of a few hundred events per year. If the mass of $W_{R}$ is close to its present lower limit $650 \mathrm{GeV}$ and we require 10 events for discovery then one year of effective LHC run will cover $M_{\Delta_{R}^{++}}$up to $2.4 \mathrm{TeV}$. The Drell-Yan pair production cross section exceeds the $W W$ fusion one only for $M_{\Delta_{R}^{++}} \lesssim 0.5 \mathrm{TeV}$ and falls below the observable limit if $M_{\Delta_{R}^{++}} \gtrsim 1$ $\mathrm{TeV}$.

Single $\Delta_{L}^{++}$production can take place if $v_{L}$ is non-zero. With the maximal presently allowed value $v_{L}=9 \mathrm{GeV}$ the LHC discovery reach during one year run extends up to 1.75 TeV, while the corresponding limit in Drell-Yan process is $1 \mathrm{TeV}$. Note that in this case $\Delta_{L}^{++}$is expected to be somewhat lighter than $\Delta_{R}^{++}$which makes the $W W$ fusion process especially interesting to search for. In conclusion, with the present constraints on the model parameters the $\Delta^{++}$masses achievable in $W W$ fusion processes exceed by a factor of two the ones testable in Drell-Yan pair production.

In the accessible mass range the decay of $\Delta_{R}$ is dominated by the channel $\Delta_{R}^{++} \rightarrow$ $l^{+} l^{+}$, providing an excellent signal. This decay provides interesting information, because its amplitude measures the strength of the lepton number violating Yukawa coupling, which plays a central part in the see-saw mechanism of neutrino masses. Depending on the respective masses, the decay modes $\Delta_{R}^{++} \rightarrow W_{R}^{+} W_{R}^{+}$and $\Delta_{R}^{++} \rightarrow W_{R}^{+} h^{+}$may also contribute. In the case of the left-triplet Higgs boson the decay $\Delta_{L}^{++} \rightarrow W_{L}^{+} W_{L}^{+}$, which can be detected via $W_{L}$ hadronic decay channels, may dominate over the decay $\Delta_{L}^{++} \rightarrow l^{+} l^{+}$.

Acknowledgements We thank R. Vuopionperä for discussions. J.M. gratefully acknowledges a grant from Jenny ja Antti Wihurin rahasto and M.R. a post doctoral grant 
from Spanish Ministry of Science and Education. This work has been supported by the Academy of Finland, Turun Yliopistosäätiö, and CICYT, grant AEN-93-0234.

\section{References}

[1] For an overview see, F. Cuypers and S. Davidson, hep-ph/9609487, submitted to Phys. Rep.

[2] J.C. Pati and A. Salam, Phys. Rev. D 10 (1974) 275;

R.N. Mohapatra and J. C. Pati, Phys. Rev. D 11 (1975) 566, 2558;

G. Senjanovic and R. N. Mohapatra, Phys. Rev. D 12 (1975) 1502;

R. N. Mohapatra and R. E. Marshak, Phys. Lett. 91 B (1980) 222.

[3] M. Gell-Mann, P. Ramond and R. Slansky, in Supergravity, eds. P. van Niewenhuizen and D. Z. Freedman (North Holland 1979);

T. Yanagida, in Proceedings of Workshop on Unified Theory and Baryon Number in the Universe, eds. O. Sawada and A. Sugamoto (KEK 1979).

[4] See e.g., B.T. Cleveland et al., in the Proceedings of the 16th International Conference on Neutrino Physics and Astrophysics, eds. A. Dar, G. Eilam and M. Gronau, Eilat, Israel 29 May - 3 June 1994, Nucl. Phys. B (Suppl.) 38 (1995) 47; Y. Suzuki, ibid. 54; J.N. Abdurashitov et al., ibid. 60; P. Anselmann et al., ibid. 68.

[5] K.S. Hirata et al., Phys. Lett. B 280 (1992) 146;

D. Casper et al., Phys. Rev. Lett. 66 (1993) 2561.

[6] G.F. Smoot et al., Ast. J. 396 (1992) L1.

[7] F. Abe et al., CDF Collaboration, Phys. Rev. Lett. 68 (1992) 1463.

[8] S. Abachi et al., D0 Collaboration, Phys. Rev. Lett. 76 (1996) 3271.

[9] Particle Data Group, Review of Particle Physics, Phys. Rev. D 54 (1996) 1.

[10] P. Langacker and S. Uma Sankar, Phys. Rev. D 40 (1989) 1569;

T. Rizzo, Phys. ReV. D 50 (1994) 325, and ibid. 5602;

G. Barenboim, J. Bernabeu, J. Prades and M. Raidal, hep-ph/9611347, submitted to Phys. Rev. D.

[11] T. Rizzo, Phys. Lett. 116 B (1982) 23; D. London, G. Belanger and J.N. Ng, Phys. Lett. B 188 (1987) 155; J. Maalampi, A. Pietilä and J. Vuori, Nucl. Phys. B 381 
(1992) 544, and Phys. Lett. B 297, 327 (1992); J. Maalampi and A. Pietilä, Z. Physik C 59 (1993) 257; C. A. Heusch and P. Minkowski, Nucl. Phys. B 416, 3 (1994); K. Huitu, J. Maalampi and M. Raidal, Nucl. Phys. B 420 (1994) 449, and Phys. Lett. B 328 (1994) 60; J.Gluza and M. Zrałek, Phys. Rev. D 52 (1995) 6238; J.Gluza and M. Zrałek, Phys. Lett. B 362 (1995) 148; P. Helde, K. Huitu, J. Maalampi and M. Raidal, Nucl. Phys. B 437 (1995) 305; A. Pietilä and J. Maalampi, Phys. Rev. D 52 (1995) 1386.

[12] J.F. Gunion, Int. J. Mod. Phys. A 11 (1996) 1551.

[13] E. Accomando and S. Petrarca, Phys. Lett. B 323 (1994) 212.

[14] J.A. Grifols, A. Mendez and G.A. Schuler, Mod. Phys. Lett. A 4 (1989) 1485;

J.F. Gunion, J. Grifols, A. Mendez, B. Kayser, and F. Olness, Phys. Rev. D 40 (1989) 1546.

[15] D. Dicus and R. Vega, Nucl. Phys. B 329 (1990) 533.

[16] M.L. Swartz, Phys. Rev. D 40 (1989) 1521;

M. Lusignoli and S. Petrarca, Phys. Lett. B 226 (1989) 397;

R. Mohapatra, Phys. Rev. D 46 (1992) 2990.

[17] R. Mohapatra, Phys. Rev. D 34 (1986) 909.

[18] N.G. Deshpande, J.F. Gunion, B. Kayser, and F. Olness, Phys. Rev. D 44 (1991) 837.

[19] H. Plothow-Besch, Comp. Phys. Comm. 75 (1993) 396;

H. Plothow-Besch, Int. J. Mod. Phys. A 10 (1995) 2901.

[20] G.P. Lepage, J. Comp. Phys. 27 (1978) 192.

[21] M. Cvetic, P. Langacker and B. Kayser, Phys. Rev. Lett. 68 (1992) 2871.

[22] V. Barger, K. Cheung, T. Han, R.J.N. Phillips, Phys. Rev. D 42 (1990) 3052.

[23] J. Lindfors, Z. Phys. C 28 (1985) 427.

[24] See P. Helde et al. in reference [11]. 